\title{
QCDMPI on Knoppix
}

\section{Shinji Hioki}

Tezukayama University

Tezukayama 7-1-1, Nara, 631-8501, JAPAN

E-mail: hiokiltezukayama-u.ac.jp

QCDMPI is a Public Domain Software. One can perform the QCD simulation on the parallel computer without any special knowledge by using QCDMPI. QCDMPI, however, requires MPI platform. This makes difficult for beginners to start the QCD simulation because they don't have any parallel computers or any parallel platform.

"QCDMPI on Knoppix" does not require any special environment; it only requires computers connected with a network.

One can get the physical results easily by the use of this package.

The XXV International Symposium on Lattice Field Theory

Regensburg, Germany

July 30 -August 4, 2007 


\section{QCDMPI}

QCDMPI[1] is a Public Domain Software. One can perform the QCD simulation on the parallel computer without any special knowledge by using QCDMPI.

One can get source codes from the QCDMPI Web Page[2] and can easily make the executable files with appropriate options.

When one wants to run QCDMPI on a single computer, one can get the result as follows:

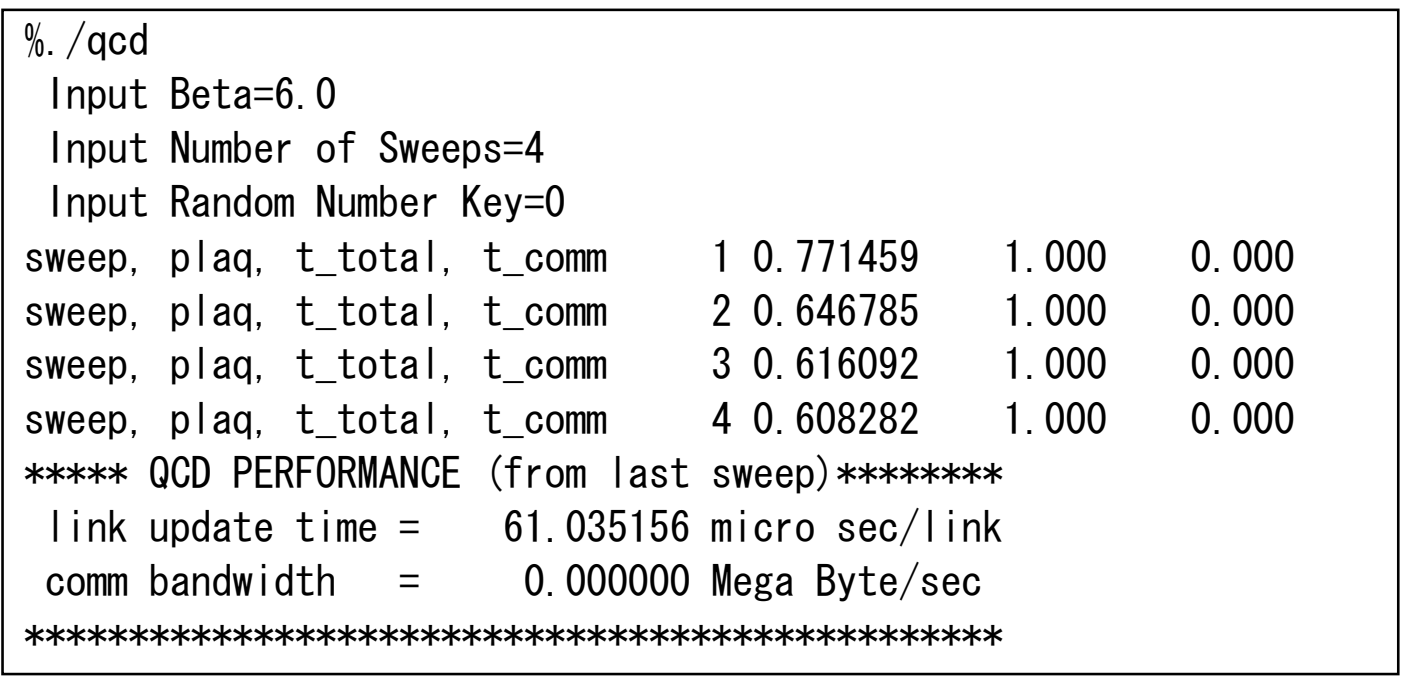

When one wants to run QCDMPI on a parallel computer, one can easily set the parameters appropriately, and can get the result as follows:

\begin{tabular}{|l} 
\%mpirun -np 8 qcd \\
Input Beta=6. 0 \\
Input Number of Sweeps=4 \\
Input Random Number Key=0
\end{tabular}

One can obtain the physical value ( plaquette value), the total performance ( link update time ) and the bandwidth of the network ( Mega Byte / sec ).

Benchmark results on several computers can be seen on the QCDMPI Web Page [2].

QCDMPI is very portable because it runs on many types of parallel computers supporting MPI. However, when one can not access MPI environment, QCDMPI does not work at all. 


\section{QCDMPI on Knoppix}

Knoppix[3] is a software based on "Linux", which is written on a CD-ROM as a bootable image.

"QCDMPI on Knoppix" is an extention of this package to QCD. It enables the beginners to perform QCD simulation without any parallel platform. It only requires computers connected with a network.

In order to perform QCD simulation with this package;

1) boot a $\mathrm{PC}$ in terms of this $\mathrm{CD}(\mathrm{DVD})$

2) boot PCs using PXE ( in terms of the network )

3) input QCD parameters ( beta, lattice size, etc )

4) get outputs ( plaquette value, bandwidth, etc )

\begin{tabular}{|l} 
\%. /QCDonKnoppix \\
Input Beta=6. \\
Input Number of Sweeps=4 \\
Input Random Number Key=0 \\
Input number of processors=8
\end{tabular}

With this package, We hope that QCD parallel simulation becomes easy to perform for beginners and we hope a lot of young researchers come into this field.

\section{References}

[1] S. Hioki, Nucl.Phys.B(Proc.Suppl.)42(1995)870-872.

S. Hioki, Parallel Computing, 22-10(1996)1335-1344.

[2] S. Hioki, QCDMPI HomePage, http://insam.sci.hiroshima-u.ac.jp/QCDMPI/

[3] Knoppix Web Page, http://www.knoppix.net/ 